\title{
Orion Optical Navigation Progress Toward Exploration Mission 1
}

\author{
Greg N. Holt*, Christopher N. D’Souza ${ }^{\dagger}$, and David Saley ${ }^{\ddagger}$ \\ NASA Johnson Space Center, 2101 NASA Parkway, Houston, TX, 77058
}

\begin{abstract}
Optical navigation of human spacecraft was proposed on Gemini and implemented successfully on Apollo as a means of autonomously operating the vehicle in the event of lost communication with controllers on Earth. It shares a history with the "method of lunar distances" that was used in the 18th century and gained some notoriety after its use by Captain James Cook during his 1768 Pacific voyage of the HMS Endeavor. The Orion emergency return system utilizing optical navigation has matured in design over the last several years, and is currently undergoing the final implementation and test phase in preparation for Exploration Mission 1 (EM-1) in 2019. The software development is being worked as a Government Furnished Equipment (GFE) project delivered as an application within the Core Flight Software of the Orion camera controller module. The mathematical formulation behind the initial ellipse fit in the image processing is detailed in Christian. ${ }^{2}$ The non-linear least squares refinement then follows the technique of Mortari ${ }^{1}$ as an estimation process of the planetary limb using the sigmoid function. The Orion optical navigation system uses a body fixed camera, a decision that was driven by mass and mechanism constraints. The general concept of operations involves a 2 hour pass once every 24 hours, with passes specifically placed before all maneuvers to supply accurate navigation information to guidance and targeting. The pass lengths are limited by thermal constraints on the vehicle since the OpNav attitude generally deviates from the thermally stable tail-to-sun attitude maintained during the rest of the orbit coast phase. Calibration is scheduled prior to every pass due to the unknown nature of thermal effects on the lens distortion and the mounting platform deformations between the camera and star trackers. The calibration technique is described in detail by Christian, et al. ${ }^{3}$ and simultaneously estimates the Brown-Conrady coefficients and the Star Tracker/Camera interlock angles. Accurate attitude information is provided by the star trackers during each pass. Figure 1 shows the various phases of lunar return navigation when the vehicle is in autonomous operation with lost ground communication. The midcourse maneuvers are placed to control the entry interface conditions to the desired corridor for safe landing. The general form of optical navigation on Orion is where still images of the Moon or Earth are processed to find the apparent angular diameter and centroid in the camera focal plane. This raw data is transformed into range and bearing angle measurements using planetary data and precise star tracker inertial attitude. The measurements are then sent to the main flight computer's Kalman filter to update the onboard state vector. The images are, of course, collected over an arc to converge the state and estimate velocity. The same basic technique was used by Apollo to satisfy loss-of-comm, but Apollo used manual crew sightings with a vehicle-integral sextant instead of autonomously processing optical imagery. The software development is past its Critical Design Review, and is progressing through test and certification for human rating. In support of this, a hardware-in-the-loop test rig was developed in the Johnson Space Center Electro-Optics Lab to exercise the OpNav system prior to integrated testing on the Orion vehicle. Figure 2 shows the rig, which the test team has dubbed OCILOT (Orion Camera In the Loop Optical Testbed). Analysis performed to date shows a delivery that satisfies an allowable entry corridor as shown in Figure 3.
\end{abstract}

*NASA Orion Navigation Lead, Aeroscience and Flight Mechanics Division, EG6, AIAA Senior Member

${ }^{\dagger}$ Navigation Technical Discipline Lead, Aeroscience and Flight Mechanics Division, EG6, AIAA Associate Fellow

$\ddagger$ Orion Navigation Hardware Subsystem Manager, Aeroscience and Flight Mechanics Division, EG2, AIAA Member 


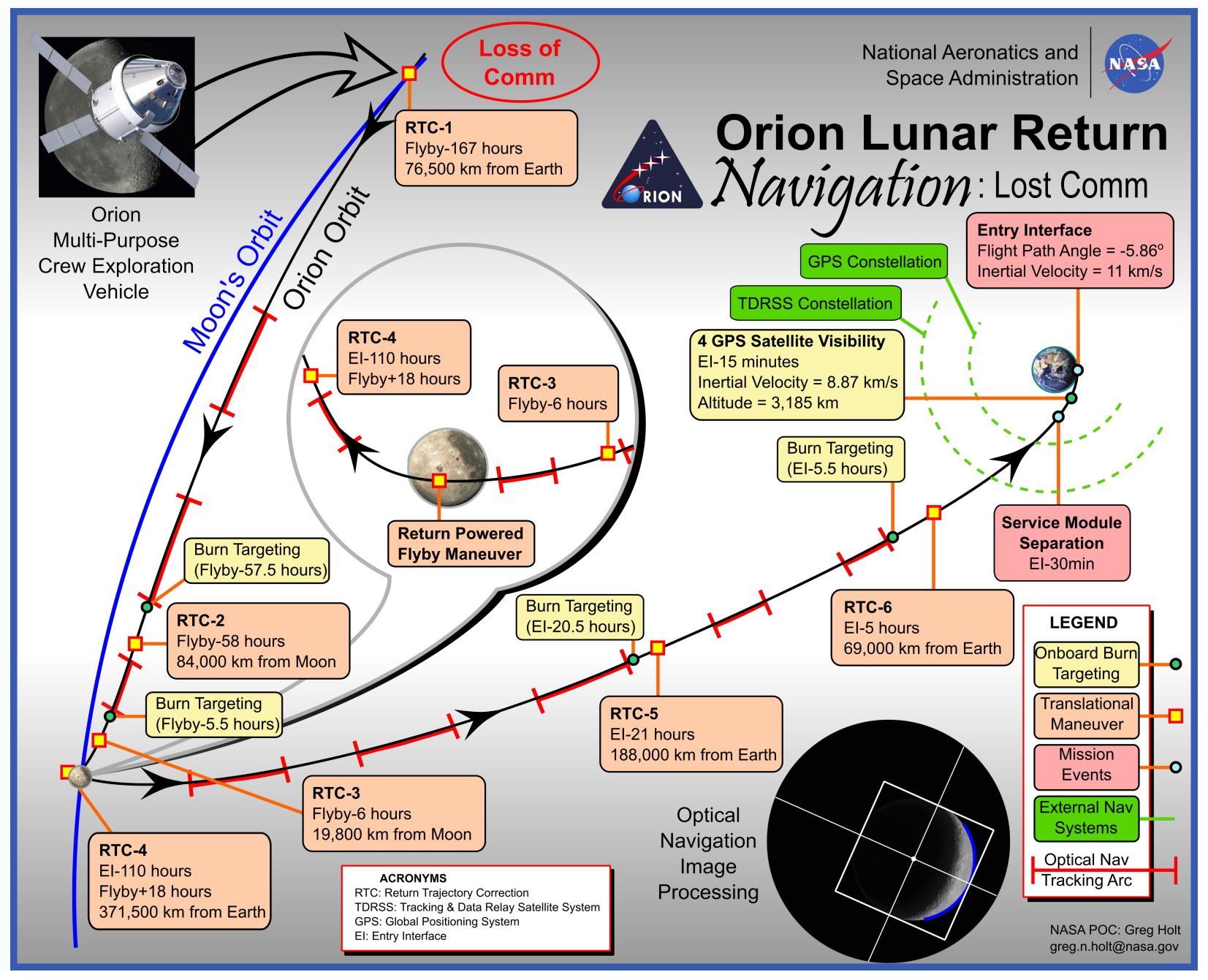

Figure 1. Orion Lunar Return Navigation Concept for Loss Of Communications 


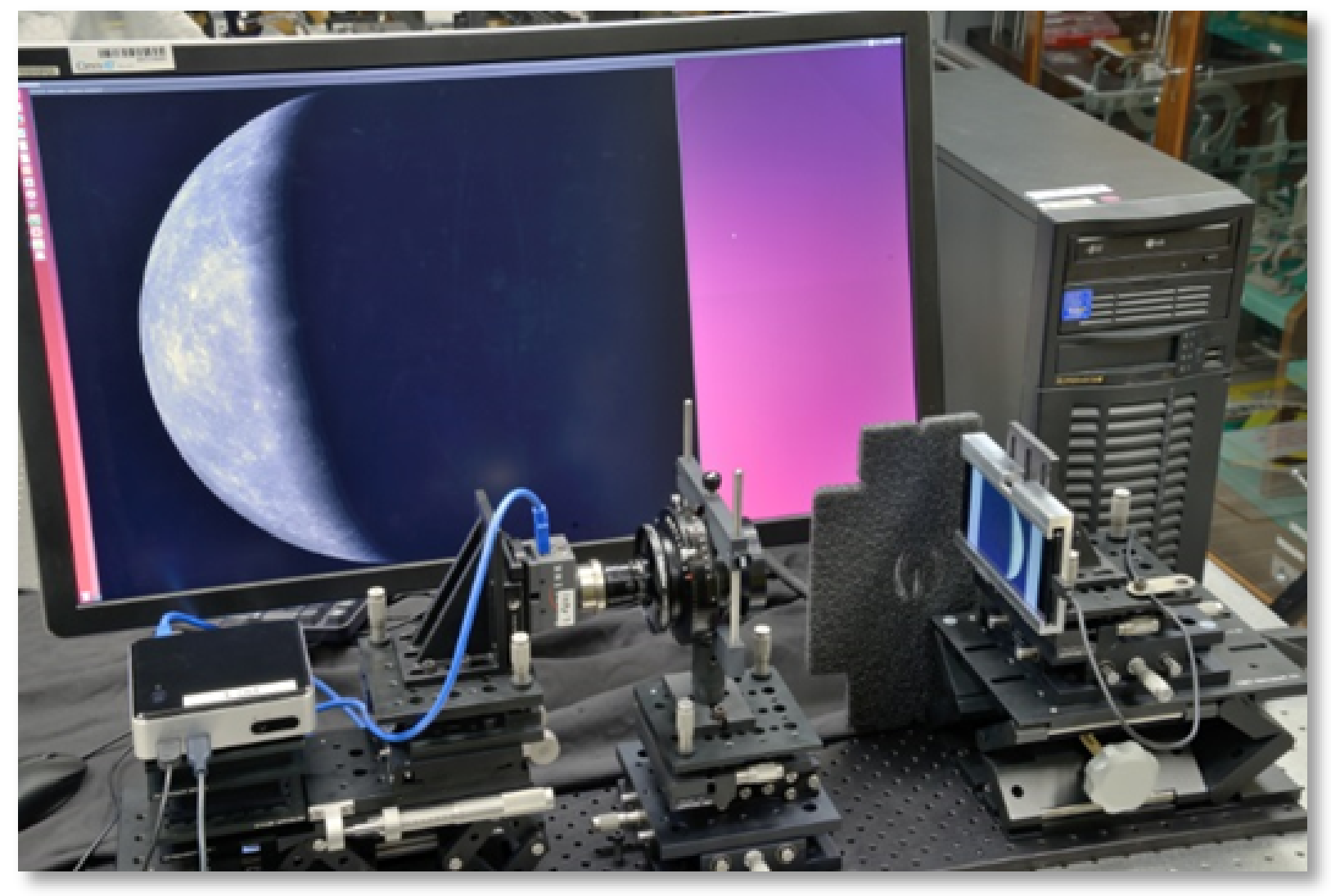

Figure 2. Orion Camera In the Loop Optical Testbed (Photo courtesy NASA/Steve Lockhart) 


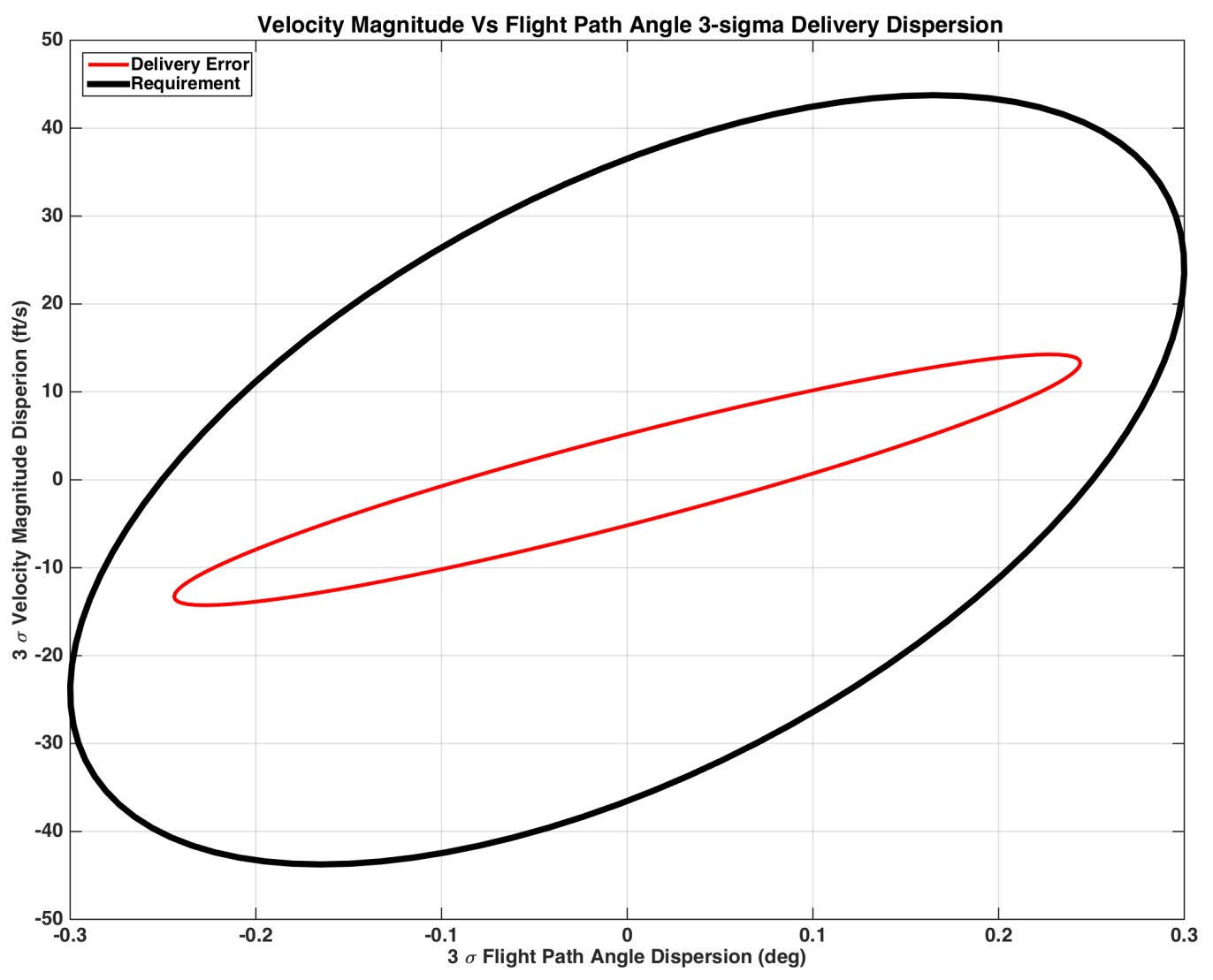

Figure 3. Orion Entry Interface Delivery Dispersions vs. Corridor Requirement 


\section{References}

${ }^{1}$ Mortari, Daniele, C. N. D'Souza, and R. Zanetti, "Image Processing of Illuminated Ellipsoid", Journal of Spacecraft and Rockets, Vol. 53, No. 3, May-June 2016.

${ }^{2}$ Christian, John A., "Optical Navigation Using Planet's Centroid and Apparent Diameter in Image", AIAA Journal of Guidance, Control, and Dynamics, Vol. 38, No. 2, Feb 2015.

${ }^{3}$ Christian, John A., Benhacine, L., and Hikes, J. et al., "Geometric Calibration of the Orion Optical Navigation Camera Using Star Field Images, The Journal of the Astronautical Sciences, Vol. 63, No. 4, Dec. 2016. 